\title{
Near real-time ionospheric monitoring over Europe at the Royal Observatory of Belgium using GNSS data
}

\author{
Nicolas Bergeot*, Jean-Marie Chevalier, Carine Bruyninx, Eric Pottiaux, Wim Aerts, Quentin Baire, \\ Juliette Legrand, Pascale Defraigne, and Wei Huang \\ Royal Observatory of Belgium, 3 avenue Circulaire, 1180 Brussels, Belgium \\ *Corresponding author: nicolas.bergeot@oma.be
}

Received 21 February 2014 / Accepted 15 September 2014

\begin{abstract}
Various scientific applications and services increasingly demand real-time information on the effects of space weather on Earth's atmosphere. In this frame, the Royal Observatory of Belgium (ROB) takes advantage of the dense EUREF Permanent GNSS Network (EPN) to monitor the ionosphere over Europe from the measured delays in the GNSS signals, and provides publicly several derived products. The main ROB products consist of ionospheric vertical Total Electron Content (TEC) maps over Europe and their variability estimated in near real-time every $15 \mathrm{~min}$ on $0.5^{\circ} \times 0.5^{\circ}$ grids using GPS observations. The maps are available online with a latency of $\sim 3 \mathrm{~min}$ in IONEX format at ftp://gnss.oma.be and as interactive web pages at www.gnss.be. This paper presents the method used in the ROB-IONO software to generate the maps. The ROB-TEC maps show a good agreement with widely used post-processed products such as IGS and ESA with mean differences of $1.3 \pm 0.9$ and $0.4 \pm 1.6 \mathrm{TECu}$ respectively for the period 2012 to mid-2013. In addition, we tested the reliability of the ROB-IONO software to detect abnormal ionospheric activity during the Halloween 2003 ionospheric storm. For this period, the mean differences with IGS and ESA maps are $0.9 \pm 2.2$ and $0.6 \pm 6.8 \mathrm{TECu}$ respectively with maximum differences $(>38 \mathrm{TECu}$ ) occurring during the major phase of the storm. These differences are due to the lower resolution in time and space of both IGS and ESA maps compared to the ROBTEC maps. A description of two recent events, one on March 17, 2013 and one on February 27, 2014 also highlights the capability of the method adopted in the ROB-IONO software to detect in near real-time abnormal ionospheric behaviour over Europe. In that frame, ROB maintains a data base publicly available with identified ionospheric events since 2012 .
\end{abstract}

\section{Introduction}

One of the main perturbations encountered by Global Navigation Satellite Systems (GNSS) signals when traveling from the satellite to the Earth is the ionospheric refraction. As the ionosphere is a dispersive medium, the ionospheric refraction depends on the signal frequency. Thus, the combination of GNSS measurements on two separate frequencies allows determining the ionospheric delay between a ground receiver and a satellite. This delay is function of the integrated number of electrons encountered in the ionosphere along the signal ray path, called the Total Electron Content (TEC). It is thus possible to build ionospheric maps representing the vertical TEC (vTEC) as a function of latitude, longitude and time from the observations of a network of GNSS stations.

Several agencies are routinely monitoring the vTEC using GNSS data. Derived maps are available with different latencies (i.e. minutes to days), area extents (i.e. local to global), grid resolutions (i.e. few degrees to lower than $1^{\circ}$ ) and time scales (i.e. few minutes to hours). The monitoring can be done in post-processing such as by the IGS which combines Global Ionospheric Maps (GIMs) from CODE, ESA, JPL and UPC (Hernández-Pajares et al. 2009) or by Noveltis for the European region (SPECTRE project; Crespon et al. 2007). The ionosphere is also monitored in near real-time (NRT) by DLR within the research project SWACI (GIMs and European region; Jakowski et al. 2011), JPL (GIMs; Mannucci et al. 1998) or NOAA/SWPC (US region; Fuller-Rowell et al. 2006). The maps from these different agencies are displayed on webpages and/or distributed in ASCII data files. The most widely used ASCII format is the IONosphere Map EXchange Format files (IONEX; Schaer et al. 1998) adopted as a convention by the IGS Analysis Centres (ACs). Presently, none of the agencies mentioned above provide publicly available highresolution near real-time ionospheric maps over Europe in the IONEX format (nor ASCII).

In the frame of the Solar-Terrestrial Centre of Excellence (STCE), the Royal Observatory of Belgium (ROB) generates routinely NRT ionospheric maps over Europe since the beginning of 2012. This product completes the space weather monitoring effort at the STCE ranging from the Sun to the upper part of the Earth atmosphere. The goal is to monitor in NRT the ionospheric state over Europe and to distribute publicly the results. The ROB-TEC maps are used by several ionospheric web services such as the European Digital Upper Atmosphere Server (DIAS; Belehaki et al. 2007) supported by the European Commission, and for several scientific studies (Sotomayor-Beltran et al. 2013; Tsagouri et al. 2013). The present paper proposes a complete description of the method developed at ROB for the ionospheric monitoring over Europe as well as a validation of its derived products.

The paper starts with a presentation of the processing strategy developed in the ROB-IONO software for retrieving vTEC over Europe every $15 \mathrm{~min}$ and detecting abnormal ionospheric activity in NRT using the GPS data from the EUREF permanent network (EPN; Bruyninx et al. 2012). The estimation of vTEC maps requires accounting for some inter-frequency hardware delays in the satellites as well as in the ground 
stations commonly named Differential Code Biases (DCBs). In the second part of the paper, the ground station DCBs estimated during the processing of NRT ROB-TEC maps are compared to the post-processed products from IGS (Hernández-Pajares et al. 2009) and ESA (Feltens 2007), which are considered as references for many ionospheric and geophysical studies (e.g. Bergeot et al. 2013; Mukhtarov et al. 2013). Finally, the effectiveness of ROB-IONO software to detect disturbances in the ionospheric state during space weather events is demonstrated.

\section{Near real-time ionospheric monitoring: ROB-IONO Software}

The main ionospheric parameter derived from GNSS measurements is the TEC expressed in TEC units $(1 \mathrm{TECu}=$ $10^{16} \mathrm{e}^{-} \mathrm{m}^{-2}$ ). For example, $10 \mathrm{TECu}$ induces a delay of $5.4 \mathrm{~ns}(=1.6 \mathrm{~m})$ on the travel time of L1 signals, and a delay of $8.9 \mathrm{~ns}(=2.7 \mathrm{~m})$ on the travel time of L2 signals. The slant TEC (sTEC) is the integrated number of electrons encountered along a satellite-receiver line of sight expressed in TECu. The sTEC between a satellite $i$ and a receiver $p$ can be retrieved using the geometry-free combination of code measurements on two different frequencies. To date, only measurements from the GPS constellation are used in our processing. The sTEC is thus estimated from the geometry-free combination of the $\mathrm{P}(\mathrm{Y})$-codes on $\mathrm{L} 1$ and $\mathrm{L} 2$ recorded by a receiver $p$ from the satellite $i$ (e.g. Hernández-Pajares et al. 2007; Bergeot et al. 2011) as follows:

$$
\begin{aligned}
(\operatorname{sTEC})_{p}^{i}= & \frac{f_{1}^{2} \cdot f_{2}^{2}}{40.3\left(f_{1}^{2}-f_{2}^{2}\right)} \\
& \times\left\{\left[\left(\tilde{P}_{2}\right)_{p}^{i}-\left(\tilde{P}_{1}\right)_{p}^{i}\right]-c \cdot\left[\mathrm{DCB}_{p}+\mathrm{DCB}^{\mathrm{i}}\right]\right\},
\end{aligned}
$$

where the sTEC depends on the two GPS carrier frequencies $f_{1}$ and $f_{2}$, the phase-smoothed code observables $\tilde{P}_{2}$ and $\tilde{P}_{1}$, the DCBs of the satellite $i$ and receiver $p$. The DCBs are the differences between the hardware delays of the signal $\mathrm{P}_{1}$ and $\mathrm{P}_{2}$ (Coco et al. 1991; Sardón et al. 1994). Satellite DCBs represent the frequency-dependent signal delays in the satellite hardware, while receivers DCBs represent the frequencydependent signal delays at the ground station (antenna and receiver). Satellite and receiver DCBs are known to be the main errors in the estimation of sTEC using GPS data with a combined effect of the order of $10 \mathrm{~ns}$. If not taken into account the DCBs would lead to an error larger than 20 TECu in the estimation of sTEC. It is therefore necessary to accurately estimate these DCBs and to reduce the correlation with the sTEC estimation in Eq. (1). The approach used here is to separate the determination of the DCBs from the sTEC estimation.

While the satellite DBCs are routinely available from IGS ACs, the estimation of receiver DCBs for all the stations of the EPN delivering data in NRT is however a critical point in our NRT ionospheric modelling. Many methods are available in the literature for estimating receiver DCBs. Two options are especially drawn from previous studies. The first one is to estimate simultaneously the sTEC and the DCBs (e.g. Schaer et al. 1998; Crespon et al. 2007) using constraints on the evolution of the ionospheric behaviour and on the DCBs. However, this option is not adequate to detect abnormal ionospheric behaviour in NRT due to the heavy constraints applied on the ionospheric behaviour and/or on the DCBs values to decrease their high correlation (e.g. Sardón \& Zarraoa 1997) and is more dedicated to a post processing approach due to a lack of observations in NRT. The second option is to use an a priori ionospheric model for estimating independently the DCBs from the sTEC (e.g. Sardón \& Zarraoa 1997; Jakowski et al. 2011). With this latter option, which is the one chosen here, it is required to pay particular attention to the $a$ priori model used as input.

In the ROB-IONO software, the processing is divided into two separate steps in order to deliver ionospheric maps over Europe. The first step is to estimate the receiver DCBs with a rapid post-processing strategy using an a priori ionospheric model based on observed data. The second step is to estimate the VTEC in NRT using the EPN network in order to deliver the ROB-TEC maps over Europe in both image and IONEX formats.

\section{Step 1: Receiver DCBs estimation in rapid post-processing}

Previous studies have shown that DCBs variations between consecutive days are smaller than $0.5 \mathrm{~ns}(<1 \mathrm{TECu})$ for the GPS satellites and smaller than $1 \mathrm{~ns}(<2 \mathrm{TECu})$ for the GPS stations (e.g. Sardón \& Zarraoa 1997). Taking into account these small variations, we choose to use the receiver and satellite DCBs estimated from the previous days with respect to the day of interest resulting in an independent estimation of the sTEC in NRT.

The satellite DCBs are taken from the rapid IONEX files distributed by CODE available with one-day latency. As the day-to-day satellite DCB variations are negligible, we considered that the satellite DCBs extracted from the rapid CODE products are representative for the day of interest.

The receiver DCBs are estimated using daily Receiver Independent EXchange Format (RINEX) files from the EPN and rapid CODE IONEX products as a priori ionospheric model. We choose this product because it provides a rapid (1-day latency) publicly available ionospheric model based on GNSS observations. This model is used to extract the vTEC at the Ionospheric Pierce Point (IPP, see Fig. 4 in Bergeot et al. 2011) for a given receiver-satellite pair using an ionospheric single thin layer hypothesis (Wild 1994). The vTEC is then projected as STEC, with the following mapping function:

$$
(\mathrm{vTEC})_{p}^{i}=(\mathrm{sTEC})_{p}^{i} \times \sqrt{1-\left(\frac{R}{R+H} \times \cos e\right)^{2}},
$$

where $R$ is the Earth radius, $H$ is the height of the thin layer representing the ionosphere $(H=450 \mathrm{~km})$ and $e$ is the elevation of the satellite $i$ with respect to the receiver $p$.

The sTEC is then introduced in the Eq. (1) to retrieve the receiver DCBs every $30 \mathrm{~s}$ over an entire day. In addition, an elevation cut-off of $30^{\circ}$ is applied to reduce inhomogeneity effects due to the projection and the noise of low elevation data. A daily receiver DCBs is then determined as the median value over the day. Finally, the DCB values used within our NRT ionospheric monitoring for a given station is the median of the 5 previous daily receiver DCBs to avoid outliers due to receiver maintenance or potential deficiencies in the postprocessed CODE IONEX products. 
Step 2: sTEC estimation and vTEC grid interpolation in near real-time

The ROB-TEC maps are generated using the GPS data from $\sim 110$ GNSS stations of the EPN which deliver real-time data streams via the ROB NTRIP broadcaster. To ensure robustness and redundancy, the ROB-IONO software is also capable to use as backup the high-rate RINEX of the BKG broadcaster (Weber et al. 2005), delaying the ROB-TEC results by $\sim 5 \mathrm{~min}$. Every $15 \mathrm{~min}$, the streams are concatenated in order to produce RINEX files. Each RINEX file contains code $(\mathrm{C} 1$ and P2) and phase (L1 and L2) observables of the last 15 min at a $30 \mathrm{~s}$ sampling rate. Then, the code observables $\mathrm{C} 1$ are corrected with satellite-dependent DCBs using the P1-C1 conversion files (e.g. Ray 2000, 2001) provided by CODE and estimated using the GNSS measurements from the last 30 days. The $\mathrm{P} 1(\mathrm{C} 1) / \mathrm{P} 2$ codes are then smoothed using the L1/L2 carrier phases. In order to minimize border effects, the smoothing procedure is applied on a longer data batch containing the 3 previous hours additionally to the current $15 \mathrm{~min}$. Outlier rejection and cycle slip detection are also performed at this stage, using the Melbourne-Wübbena combination (Melbourne 1985; Wübbena 1985).

All the phase-smoothed GPS code data and daily receiver DCBs estimated in step 1 are then used in order to estimate in NRT the sTEC from the geometry-free combination as in Eq. (1). For that, only GPS data with an elevation angle higher than $15^{\circ}$ are considered in order to cover as much as possible the European region. The sTECs are then projected to retrieve the vTECs at the IPPs using Eq. (2). The satellite elevation and the IPP position of each satellite-receiver pair are determined using the interpolated IGS ultra-rapid GPS orbits and the station coordinates provided on a weekly basis by the EPN Central Bureau and available from $\mathrm{ftp}$ ://epncb.oma.be.

The vTECs are estimated each $30 \mathrm{~s}$ during a $15 \mathrm{~min}$ time span meaning 30 IPPs considering a satellite-receiver pair without any data interruption. Then the median of the vTECs at IPPs are estimated for 5-min sub-intervals together with their standard deviations. This permits to reduce the data redundancy along the satellite track and to optimize the vTEC data distribution for the interpolation. As a result, three values of vTEC for each satellite-receiver pair are obtained. The standard deviations of these values (ideally 3 std are estimated per satellite-receiver pair based each on 5-min observations) are interpreted as the vTEC variability giving an indicator of the ionospheric state variations during the 15-minute time span.

The vTECs at IPPs are then interpolated using thin plate splines iterated with different degrees of smoothing and rejection levels in order to filter potential outliers. The first step consists of applying a strong coefficient of smoothing to remove the vTEC values with the largest deviations $(>20 \mathrm{TECu})$ with respect to the interpolated surface. During this iteration step about $5 \%$ of the data at IPPs are classically rejected. The final interpolation uses a softer smoothing coefficient which leaves the small variations present in the vTEC maps. In parallel, the standard deviations of the non-rejected IPPs are also interpolated using thin plate splines and a smooth coefficient to provide the so-called variability maps.

The results are $0.5^{\circ} \times 0.5^{\circ}$ vTEC and vTEC variability grids extended from $-15^{\circ}$ to $25^{\circ}$ in longitude and $35^{\circ}$ to $62^{\circ}$ in latitude every $15 \mathrm{~min}$. They are available online with a

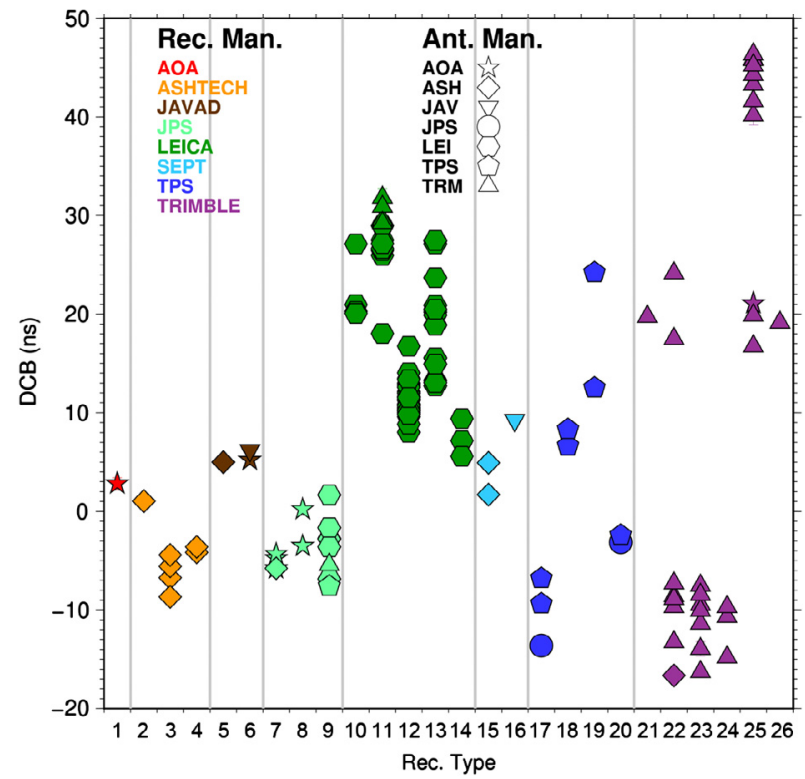

Fig. 1. Mean values of P1-P2 DCBs estimated for 117 stations of the EPN for the period 2012 to mid-2013. Each colour corresponds to a receiver manufacturer. The different receiver types produced by a given manufacturer (Rec. Type) are also taken into consideration (see Table 1). Each symbol corresponds to a specific antenna manufacturer.

latency of $\sim 3$ min on the web interface www.gnss.be, and in the IONEX format at $\mathrm{ftp}$ ://gnss.oma.be.

\section{Near real-time ROB-TEC map validation}

The validation of ROB-TEC maps is proposed at three different levels. First, the daily receiver DCBs used for the NRT ROB-TEC maps are compared with those available from the IGS and ESA. Secondly, the ROB-TEC maps are compared with the GIMs generated by IGS and ESA. Finally, the robustness of the strategy is tested by analyzing the behaviour of ROB-TEC maps during high-dynamic ionospheric activity.

\subsection{Validation of daily receiver DCBs}

Figure 1 represents the receiver P1-P2 DCBs of the 117 EPN stations estimated with the method described in the previous section and used for the NRT ROB-TEC maps. The values represented are the mean values of the P1-P2 DCBs over the period January 2012 to July 2013 (544 days) for each receiver-antenna pair (141 pairs for the period of interest). In order to emphasize the DCBs dependence on the station hardware, the stations in Figure 1 are classified as a function of their equipment.

The daily receiver DCBs obtained are ranging from -16 to 46 ns. While the receiver DCBs are relatively coherent for a given manufacturer, some discrepancies are noticeable especially for LEICA, TPS and TRIMBLE from one to another receiver type. It must also be noted that the dispersion of DCBs can be significant even for stations equipped with exactly the same receiver and antenna, as e.g. for the LEICA GRX1200+GNSS connected to a Leica antenna where the DCBs range from 13 to $28 \mathrm{~ns}$. 
Table 1. Receiver types corresponding to Figure 1.

\begin{tabular}{ccc||ccc}
\hline \hline Rec type & & Receiver & Rec type & \multicolumn{2}{c}{ Receiver } \\
\hline 1 & AOA & SNR-12 & 14 & LEICA & GRX1200PRO \\
2 & ASHTECH & UZ-12 & 15 & SEPT & POLARX2E \\
3 & ASHTECH & Z-XII3 & 16 & SEPT & POLARX4TR \\
4 & ASHTECH & Z-XII3T & 17 & TPS & E_GGD \\
5 & JAVAD & TRE_G3TDELTA & 18 & TPS & NETG3 \\
6 & JAVAD & TRE_G3THDELTA & 19 & TPS & NET-G3A \\
7 & JPS & E_GGD & 20 & TPS & ODYSSEY_E \\
8 & JPS & EGGDT & 21 & TRIMBLE & T700 \\
9 & LPS & GEACY & 22 & TRIMBLE & NETR5 \\
10 & LEICA & GRIMBLE & NETR8 \\
11 & LEICA & GR25 & 24 & TRIMBLE & NETR9 \\
12 & LEICA & GRX1200GGPRO & 25 & TRIMBLE & NETRS \\
13 & LEICA & GRX1200+GNSS & 26 & TRIMBLE & R7 \\
\hline
\end{tabular}

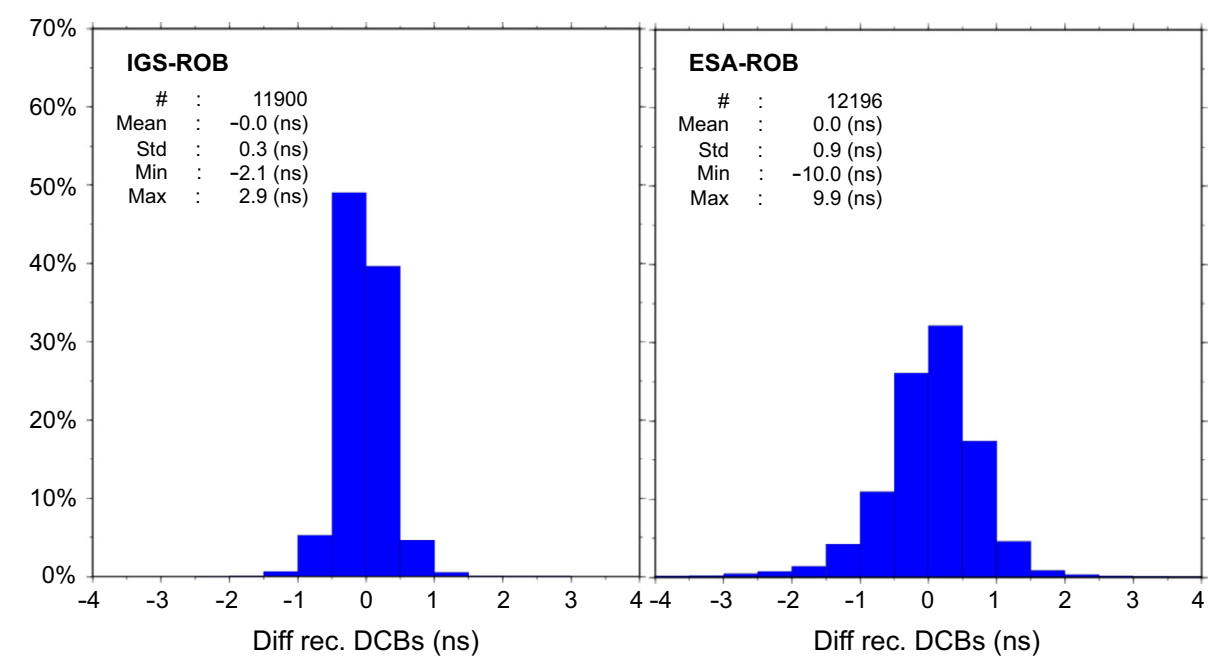

Fig. 2. Comparison of the DCBs estimated for EPN stations for the period 2012 to mid-2013 with the DCBs from IGS (left) and ESA (right) ACs. The differences are made only for common sites between ROB/IGS and ROB/ESA. The statistics of the differences are also presented.

In order to validate these receiver DCBs estimations, we compare them with IGS and ESA DCBs estimations given in their respective post-processed ionospheric products. The comparison is made on the 38 and 29 EPN stations also included in the IGS and ESA processing respectively.

The receiver DCBs used for the NRT ROB-TEC maps do not show significant biases with the post-processed estimation from IGS and ESA (Fig. 2). This confirms the reliability of the daily receiver DCBs parameters used for the realization of ROB-TEC maps in NRT. However, large discrepancies with respect to the ESA values (Fig. 2, right) are observed with important differences up to $10 \mathrm{~ns}$. While the estimated DCBs show differences with IGS below or equal to 0.5 ns for $89 \%$ of the time, this statistic decreases to $58 \%$ with ESA DCBs. As the IGS products are considered as the reference for global ionospheric monitoring, we suspect that the large discrepancies between ROB and ESA estimations are due to the strategy used by the ESA AC to retrieve these quantities. This is confirmed with the comparison between the IGS and ESA receiver DCBs. In that case, the mean difference is $0.1 \pm 0.8 \mathrm{~ns}$ for the entire period with important differences up to $9.7 \mathrm{~ns}$ which is compatible with the ROB/ESA comparison.

\subsection{Validation of ROB-TEC maps}

The NRT ROB-TEC maps are compared with the postprocessed IGS and ESA GIMs for 181 days covering the end of 2012 and the beginning of 2013. The GIMs from IGS and ESA, available in IONEX format, are estimated every $2 \mathrm{~h}$ on a $5^{\circ} \times 2.5^{\circ}$ grid (in longitude and latitude). Thus, a linear interpolation in time and space is applied on GIMs IONEX in order to match the characteristics of the ROB-TEC IONEX (i.e. $0.5^{\circ} \times 0.5^{\circ}$ grid resolution every $15 \mathrm{~min}$ ). The mean differences between the ROB and either IGS or ESA maps are presented in Figure 3.

The mean differences for the entire period are $1.3 \pm 0.9$ $\mathrm{TECu}$ in comparison with IGS and $0.4 \pm 1.6 \mathrm{TECu}$ with ESA. The bias is greater with the IGS IONEX, but larger discrepancies exist between the ROB-NRT and the ESA products, as seen from the standard deviation of the differences. In both cases, the latitudinal and longitudinal sections of the differences show the same shape (Fig. 3, right). These variations can be explained by (1) medium-scale structures only visible on the regional ROB-TEC maps, but not on the global maps which do not offer the same resolution and (2) the errors due to the linear 

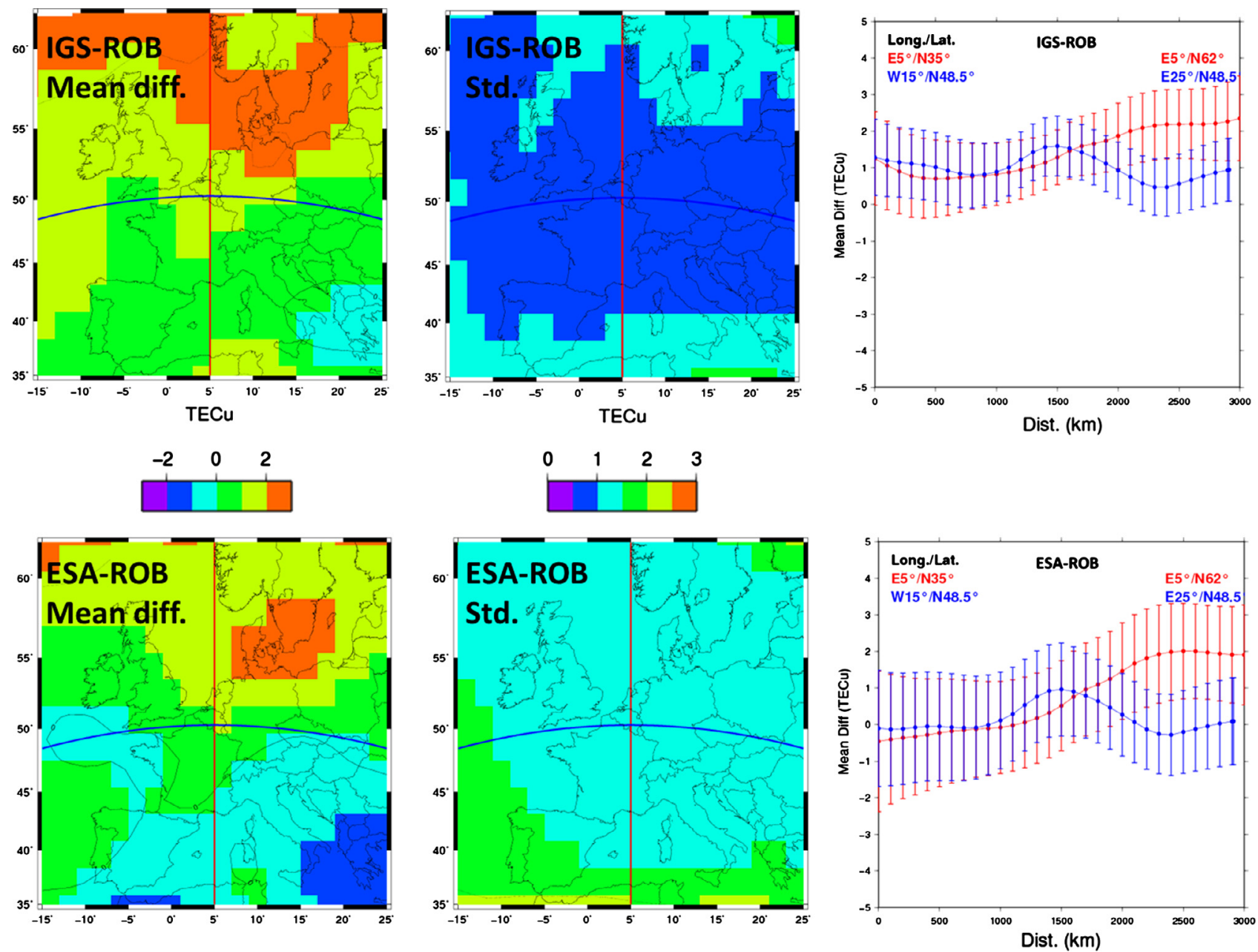

Fig. 3. Differences between NRT ROB-TEC IONEX product and post-processed IGS and ESA products, from 181 days of data covering the end of 2012 and the beginning of 2013 (17,376 maps). Left: mean differences for the entire period. Middle: standard deviation of the differences $(1 \sigma)$. Right: profile of the mean differences extracted from the maps with their standard deviation. The red (longitudinal) and blue (latitudinal) lines correspond to the two tracks shown on the maps. Top: differences between post-processed IGS and NRT ROB-TEC maps. Bottom: differences between post-processed ESA and NRT ROB-TEC maps.

interpolation applied on the GIMs to fit the ROB-TEC maps spatial and temporal resolutions. Hernández-Pajares et al. (2009) showed that the IGS and ESA products are biased by $1.0 \pm 4.4$ and $3.0 \pm 6.8 \mathrm{TECu}$ with respect to the altimeter measurements from TOPEX/JASON satellites. As noted by the authors, these differences are considered as a pessimistic estimation of the GIMs actual errors due to the lack of altimeter data over continents where the GPS-based data are more present. Consequently, the differences between the ROB-TEC maps and the post-processed GIMs are within the precision of the TEC estimation using GPS data.

\subsection{ROB-TEC maps during space weather events over Europe}

To test the capability of the ROB-IONO software to detect abnormal ionospheric activity in NRT, the software was applied on the EPN data gathered during the Halloween storm of October 28-30, 2003 considered as major geomagnetic storm which affected the Earth upper atmosphere and communications systems during the 23rd solar cycle (e.g. Mannucci et al. 2005).

Figure 4 (top left) shows the resulting ROB-TEC maps between 22:15 and 22:30 UTC. During quiet ionospheric activity, the expected vTEC values (time-series in grey on Fig. 4, right) are lower than 7 TECu during the night. However, vTEC values reached up to $47 \mathrm{TECu}$ in the northern part of the map during the night on October 30, 2003. The variability maps (Fig. 4, bottom left) highlight also high variations of vTEC within the 15 min especially in the Northern region. This is in accordance with the extreme TEC gradients occurring at mid-latitudes due to plasma increase during such event (e.g. Mannucci et al. 2005).

ROB-TEC maps agree well at first order with the IGS estimations while the differences are more scattered with respect to ESA maps. Indeed, the mean differences with IGS and ESA GIMs estimated for period 27/10/2003-01/11/2003 for three representative locations (red triangles and time series in Fig. 4) are $0.9 \pm 2.2$ and $0.6 \pm 6.8 \mathrm{TECu}$, respectively. Moreover, during the major phase of the storm (i.e. the night of October 30, 2003), differences are significant and reach up to $17 \mathrm{TECu}$ with IGS and 38 TECu with ESA estimations (Fig. 5).

This can be explained by the spatial and temporal smoothing of the ionospheric signal in IGS and ESA GIMs. Indeed, these GIMs are provided once per $2 \mathrm{~h}$ and have low spatial resolution, so that they represent only the slow changes and long wavelength structures of the ionosphere. However, no 

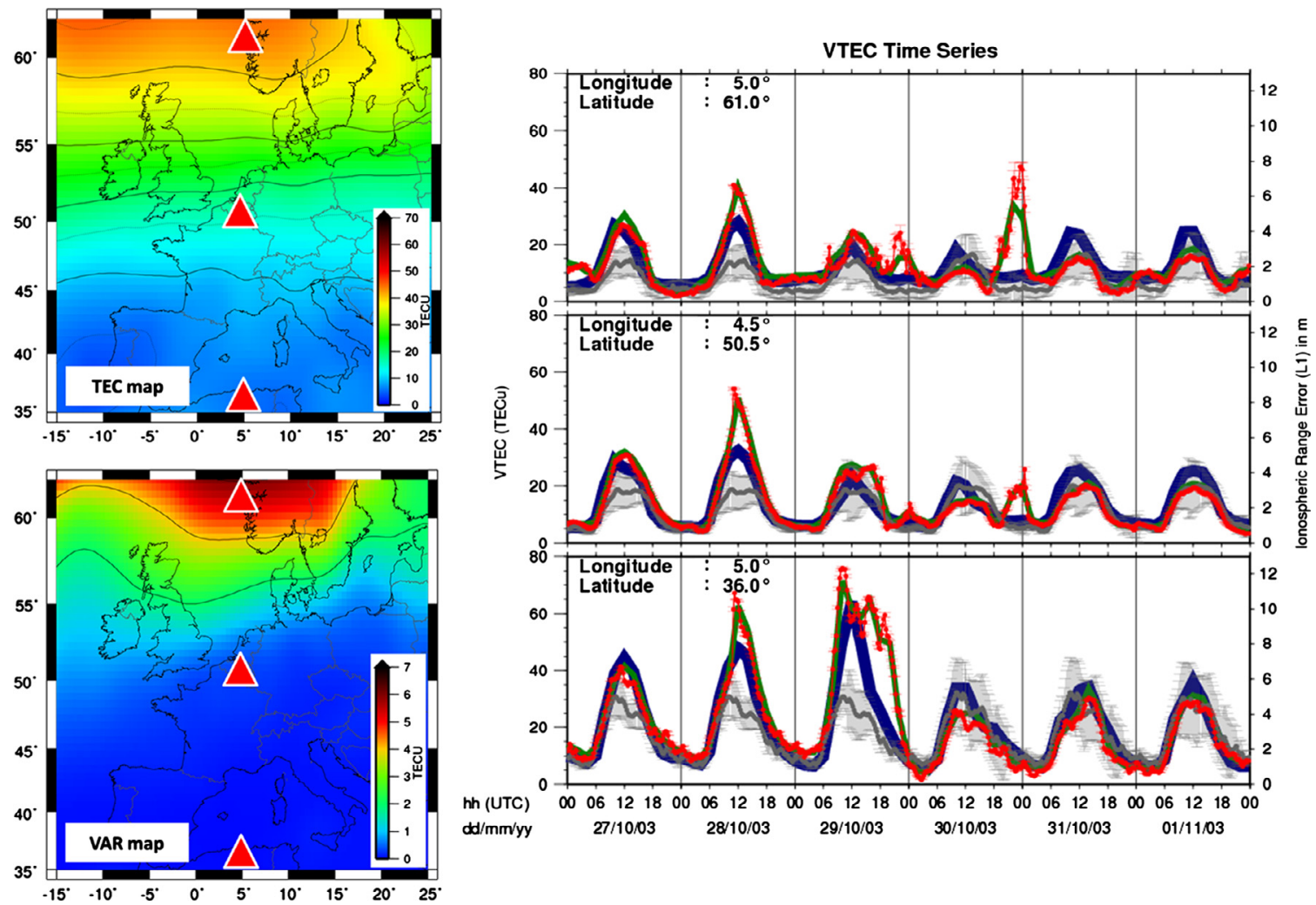

Fig. 4. NRT ROB-TEC maps during the 2003 Halloween storm. Left: ROB-TEC maps estimated between 22:15 and 22:30 UTC for October 30, 2003. Left top: ROB-TEC map estimated in NRT. Left bottom: ROB-TEC variability map during the 15-min time span. Right: 6-day vTEC time series extracted from ROB-TEC maps at three different geographic locations represented by the red triangle on the maps (top: northern part of the maps; middle: above Brussels, bottom: southern part of the maps). The red dots are the vTEC time series estimated in NRT. The grey dots represent the expected ionospheric behaviour based on the median vTEC from the 15 previous days. The green and blue lines are the interpolated values from the IGS and ESA final GIMs respectively.

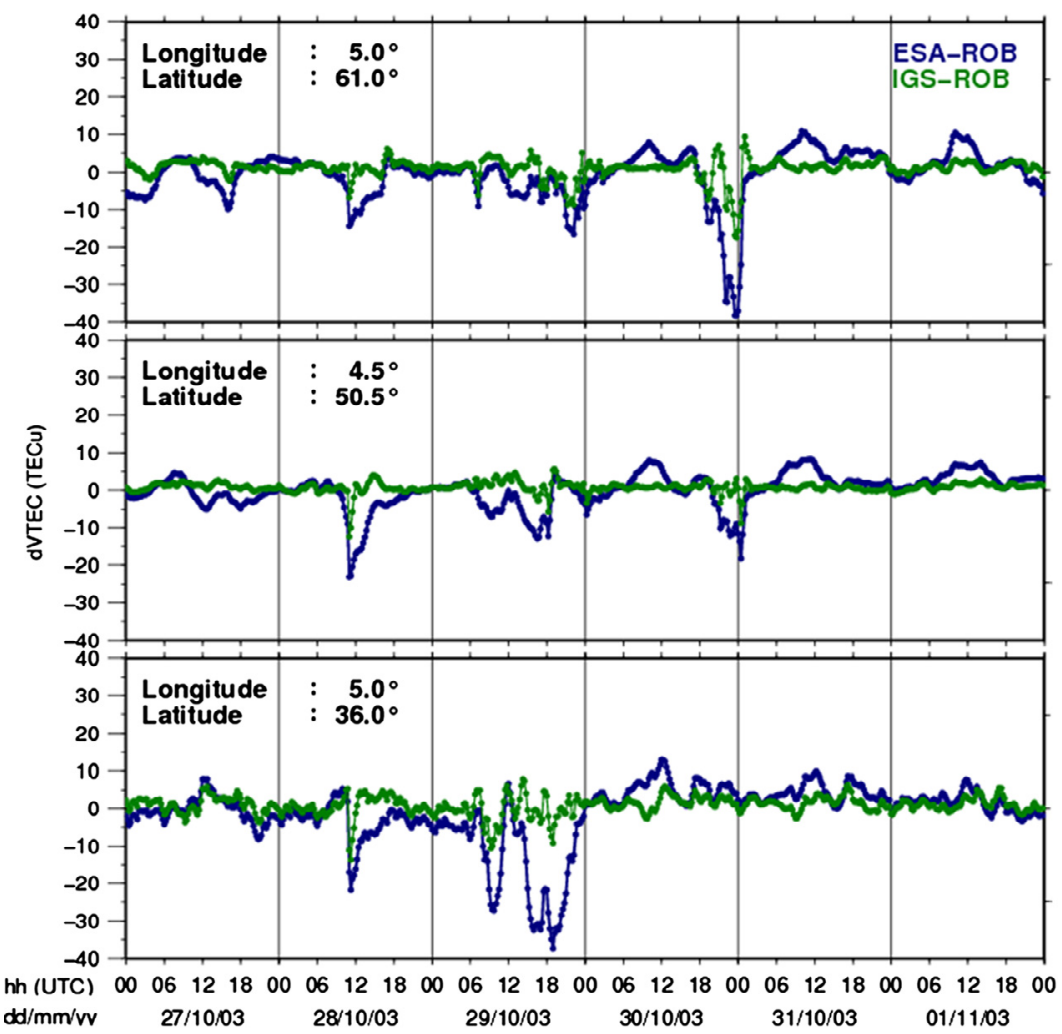

Fig. 5. Differences between ESA/IGS products and ROB-TEC maps during the 2003 Halloween storm. The 6-day time-series represent the differences between the vTEC extracted from ESA (blue) and IGS (green) maps with ROB-TEC maps at three different geographic locations shown on Figure 4. 
N. Bergeot et al.: Near real-time ionospheric monitoring over Europe at the ROB

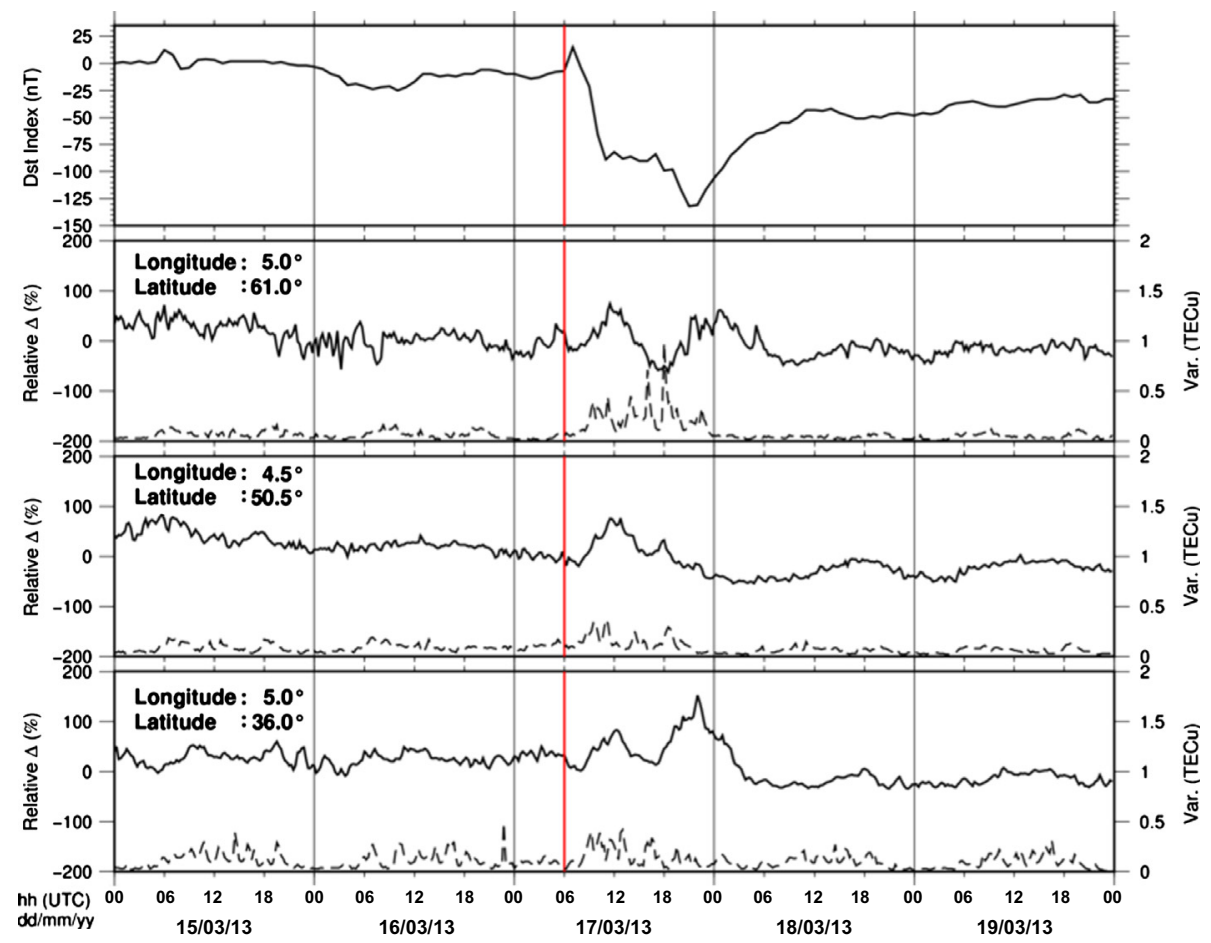

Fig. 6. Dst index and ROB-TEC products for the period March 15 to March 19, 2013. The red line is the storm onset of the intense geomagnetic storm on March 17. Top: the Dst index as delivered by the WDC Kyoto Observatory. Bottom: the three time-series extracted from ROB-TEC maps at three different geographic locations shown on Figure 4. The bold line represents the relative differences between the ROBTEC values and the median from the 15 previous days. The dashed line is the variability of the vTEC estimated in NRT.

explanation was found why the ESA AC does not notice any abnormal ionospheric activity during this major event.

Consequently, ROB-TEC IONEX together with variability maps estimated in NRT permit to follow the ionospheric behaviour in more details, bringing valuable information during a disturbed period.

\section{Discussion}

Presently, more than 30 abnormal ionospheric activity events are reported on the www.gnss.be web interface for the period 2012-2014. The space weather origin of each event is reported in collaboration with the STCE members. For the period 2012-2014, the ionospheric perturbations are associated with Coronal Mass Ejections (CMEs, $70 \%$ of the time), not well established origin of active geomagnetic conditions $(\sim 20 \%$ of the time) or unidentified phenomena $(\sim 10 \%$ of the time). To illustrate the reliability of the detection of abnormal ionospheric activity, two recent ionospheric storm events are discussed and compared with the geomagnetic Dst index delivered by the WDC Kyoto Observatory. The first intense event occurred in March 2013 (Dst $=-132$ nT, Fig. 6, top) while the second moderate event occurred in February 2014 (Dst $=-99$ nT, Fig. 7, top). For each event, the geomagnetic activity is compared with the relative differences between the ROB-TEC values estimated in NRT and the expected vTEC values (i.e. differences scaled by the 15 previous days median) as well as the estimated variability defined in Section 1 .

The March 17, 2013 ${ }^{1}$ event is concordant with a CME impact detected at 05:30 UTC by the ACE satellite with a

\footnotetext{
1 This event is reported on the web interface at this address: http://gnss.be/Atmospheric_Maps/2013-03-17
}

shock at Earth vicinity at 06:00 UTC (Baker et al. 2014). The ROB-TEC maps highlighted an abnormal increase of the vTEC with respect to the expected values at all latitudes between 09:00 and 15:00 UTC and later in the day for highand low-latitudes. The vTEC time-series extracted from ROB-TEC maps at different locations are concordant with the storm onset (Fig. 6, red line). The relative differences reach $+150 \%$ at low-latitudes (Fig. 6, bold lines on the three bottom plots) while the variability is significantly higher at high-latitudes (Fig. 6, dashed lines on the three bottom plots) with a maximum at 18:00 UTC. The mid-latitudes regions are less affected during this event. This event was detected by different space weather dedicated satellites such as the Van Allen Probes which monitor the electron particle dynamics in the Earth's radiation belt. From these data, it appears that the plasmapause boundary was forced deeply inward within the magnetosphere implying high aurora electrojet index and a recovery phase of the storm on March 18 at 03:00 UTC (Foster et al. 2014; Baker et al. 2014; Boyd et al. 2014) which is concordant with ROBTEC observations.

The second event occurred on February 27, 2014. ${ }^{2}$ During this event, the Dst decreased to $-99 \mathrm{nT}$ (see Fig. 7, top) corresponding to a moderate geomagnetic storm with an onset at 16:00 UTC. This event might be the result of the arrival of the CME associated with the solar flare event on February 25. During this period, the ROB-TEC maps detected an abnormal increase of the vTEC at high- and low-latitudes between 19:00 on February 27 and 06:00 UTC on February 28. As for the 2013 event, the mid-latitudes regions are less affected during this event. The relative differences with the expected ionospheric values exceed $+200 \%$ at low-latitudes at 22:15 UTC

\footnotetext{
${ }^{2}$ This event is reported on the web interface at this address: http://gnss.be/Atmospheric_Maps/2014-02-27
} 


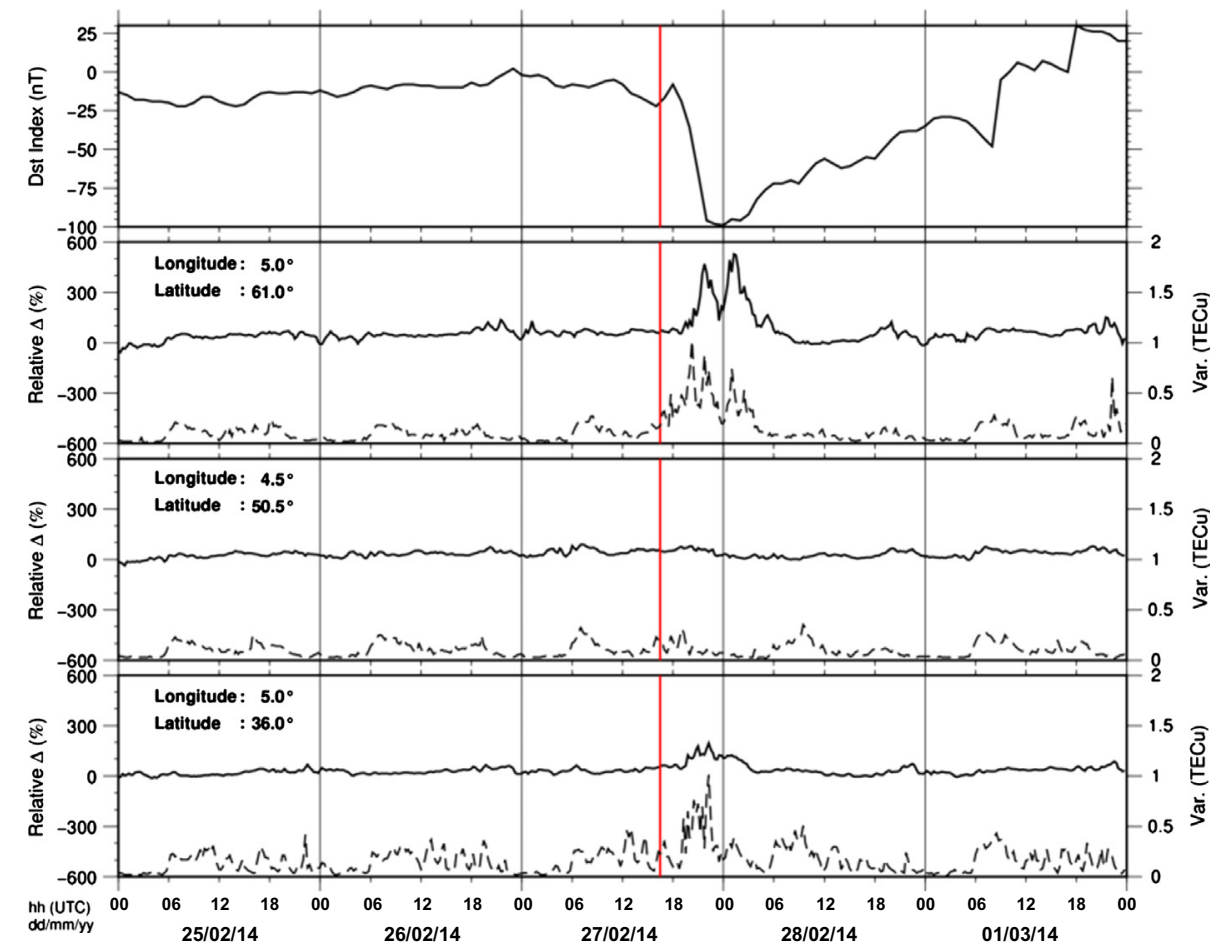

Fig. 7. Dst index and ROB-TEC products for the period February 25 to March 1, 2014. The red line is the storm onset of the moderate geomagnetic storm on February 27. Top: the Dst index as delivered by the WDC Kyoto Observatory. Bottom: the three time-series extracted from ROB-TEC maps at three different geographic locations shown on Figure 4. The bold line represents the relative differences between the ROB-TEC values and the median from the 15 previous days. The dashed line is the variability of the vTEC estimated in NRT.

and $+500 \%$ at $01: 15$ UTC at high-latitudes (Fig. 7, bold lines on the three bottom plots). It can also be noted that the vTEC variability at low- and high-latitudes (Fig. 7, dashed lines on the three bottom plots) increases slightly before the relative vTEC values and is more concordant with the storm onset detected from the Dst index. This reflects the fact that even if the ROB-TEC maps are in accordance with the expected values, the variability maps highlight the disturbances in the ionosphere linked to geomagnetic perturbations. The pre-storm conditions are retrieved few hours after the onset at midlatitudes, while it took more time for high-latitudes region. These longer perturbations at high-latitudes might be due to persistent high dynamics in the aurora region due to geomagnetically induced currents.

\section{Conclusions}

The ROB-IONO software and the method developed at the Royal Observatory of Belgium in order to retrieve in near real-time the ionospheric vTEC over Europe was introduced. The main products are $0.5^{\circ} \times 0.5^{\circ}$ grids of $\mathrm{VTEC}$ and vTEC variability available every $15 \mathrm{~min}$ with a latency of $\sim 3$ min. These maps can be consulted on a web interface (www.gnss.be) or downloaded in the IONEX format at ftp://gnss.oma.be.

The ROB-IONO software and derived near real-time ROBTEC maps were tested and validated through comparisons with widely used post-processed products such as IGS and ESA DCBs and GIMs. The receivers DCBs estimated by
ROB-IONO are in good agreement with the one provided by IGS/ESA. The differences based on $\sim 1.5$ year of data in 2012 and 2013 are of the order of $0.0 \pm 0.3 \mathrm{~ns}$ and $0.0 \pm 0.9 \mathrm{~ns}$ with respect to IGS and ESA final products respectively. The ROB-TEC maps show differences of $1.3 \pm 0.9$ and $0.4 \pm 1.6 \mathrm{TECu}$ with respect to IGS and ESA post-processed products. This is not significant in view of the current precision level of the GPS-derived TEC maps. A systematic pattern is furthermore detected, most probably due to the different methods used by ROB for the regional ionospheric map realization in comparison with IGS and ESA global processing.

The ROB-IONO software rests upon a method able to de-correlate the estimation of the DCBs and sTEC in order to follow the ionospheric disturbances in NRT. The study case of the 2003 Halloween super-storm allowed validating the ROB-TEC maps during an extreme ionospheric event. While the general trend of ROB-TEC maps is in good agreement with IGS and ESA, maximum differences can reach more than 38 TECu during high ionospheric activity. The ROB-TEC products also showed a good agreement with geomagnetic observations during less intense events such as the ones of March 17 , 2013 and February 27, 2014. Finally, the potential of the variability maps as an indicator of rapid ionospheric variations during the $15 \mathrm{~min}$ of observations is highlighted. Presently, more than 30 events during the period 2012-2014 are associated with space weather. This database is updated a few days after an identified event.

In the future, a comparison with other regional products (e.g. SWACI, SPECTRE) would give a reliable comparison 
and more information on the accuracy of such regional products. This could be done in the frame of the ESA Space Weather Situational Awareness programme aiming to provide accurate information and data on the space weather effect on near Earth environment, particularly regarding hazards to ground and in orbit-infrastructures. These maps can also serve for precise corrections of ionospheric delays in any electromagnetic signal crossing the ionosphere, as e.g. proposed by Fujieda et al. (2014) for two-way satellite frequency transfer.

Acknowledgements. This work was supported by the Solar and Terrestrial Centre of Excellence (STCE, http://www.stce.be/). The authors acknowledge the CODE AC, IGS and WDC Kyoto Observatory for providing publicly their products. We are also grateful to the BKG for the high-rate RINEX used as a backup to the ROB NTRIP broadcaster. Detailed comments and suggestions of the two reviewers and the associate editor greatly contributed to the improvement of this article. The editor thanks two anonymous referees for their assistance in evaluating this paper.

\section{References}

Baker, D.N., A.N. Jaynes, X. Li, M.G. Henderson, S.G. Kanekal, et al., Gradual diffusion and punctuated phase space density enhancements of highly relativistic electrons: Van Allen Probes observations, Geophys. Res. Lett., 41, 1351-1358, DOI: 10.1002/2013GL058942, 2014.

Belehaki, A., L. Cander, B. Zolesi, J. Bremer, C. Juren, I. Stanislawska, D. Dialetis, and M. Hatzopoulos, Ionospheric specification and forecasting based on observations from European ionosondes participating in DIAS project, Acta Geophys., 55 (3), 398-409, 2007.

Bergeot, N., C. Bruyninx, P. Defraigne, S. Pireaux, J. Legrand, E. Pottiaux, and Q. Baire, Impact of the Halloween 2003 Ionospheric Storm on Kinematic GPS Positioning in Europe, GPS Solutions, 15 (2), 171, DOI: 10.1007/s10291-010-0181-9, 2011.

Bergeot, N., I. Tsagouri, C. Bruyninx, J. Legrand, J.-M. Chevalier, P. Defraigne, Q. Baire, and E. Pottiaux, The influence of space weather on ionospheric total electron content during the 23rd solar cycle, J. Space Weather Space Clim., 3, A25, DOI: 10.1051/swsc/2013047, 2013.

Boyd, A.J., H.E. Spence, S.G. Claudepierre, J.F. Fennell, J.B. Blake, D.N. Baker, G.D. Reeves, and D.L. Turner, Quantifying the radiation belt seed population in the March 17, 2013 electron acceleration event, Geophys. Res. Lett., 41, 2275-2281, DOI: 10.1002/2014GL059626, 2014.

Bruyninx, C., H. Habrich, W. Söhne, A. Kenyeres, G. Stangl, and C. Völksen, Enhancement of the EUREF permanent network services and products, Geodesy for Planet Earth, IAG Symposia Series, 136, 27-35, DOI: 10.1007/978-3-642-20338-1_4, 2012.

Coco, D.S., C. Coker, S.R. Dahlke, and J.R. Clynch, Variability of GPS satellite differential group delay biases, IEEE Trans. Aerosp. Electron. Syst., 27 (6), 931-938, 1991.

Crespon, F., E. Jeansou, J. Helbert, G. Moreaux, P. Lognonné, P.-E. Godet, and R. Garcia, SPECTRE (www.noveltis.fr/spectre): a web service for Ionospheric Products, Proceedings of 1 st Colloquium Scientific and Fundamental Aspects of the Galileo Program, 2007.

Feltens, J., Development of a new three-dimensional mathematical ionosphere model at European Space Agency/European Space Operations Centre, Space Weather, 5, S12002,

DOI: 10.1029/2006SW000294, 2007.
Foster, J.C., P.J. Erickson, D.N. Baker, S.G. Claudepierre, C.A. Kletzing, et al., Prompt energization of relativistic and highly relativistic electrons during a substorm interval: Van Allen Probes observations, Geophys. Res. Lett., 41, 20-25,

DOI: 10.1002/2013GL058438, 2014.

Fujieda, M., D. Pieste, T. Gotoh, J. Becker, M. Aida, and A. Bauch, Carrier-phase two-way satellite frequency transfer over a very long baseline, Metrologia, 51, 253-262, DOI: 10.1088/0026-1394/51/3/253, 2014.

Fuller-Rowell, T., E. Araujo-Pradere, C. Minter, M. Codrescu, P. Spencer, D. Robertson, and A.R. Jacobson, US-TEC: a new data assimilation product from the Space Environment Center characterizing the ionospheric total electron content using realtime GPS data, Radio Sci., 41, RS6003, DOI: 10.1029/2005RS003393, 2006.

Hernández-Pajares, M., J.M. Juan, J. Sanz, R. Orús, A. Garcia-Rigo, J. Feltens, A. Komjathy, S.C. Schaer, and A. Krankowski, The IGS VTEC maps: a reliable source of ionospheric information since 1998, J. Geod., 83 (3-4), 263-275, DOI: 10.1007/s00190-008-0266-1, 2009.

Hernández-Pajares, M., J.M. Juan, J. Sanz, and R. Orús, Secondorder ionospheric term in GPS: implementation and impact on geodetic estimates, J. Geophys. Res., 112, B08417, DOI: 10.1029/2006JB004707, 2007.

Jakowski, N., C. Mayer, M.M. Hoque, and V. Wilken, Total electron content models and their use in ionosphere monitoring, Radio Sci., 46, RS0D18, DOI: 10.1029/2010RS004620, 2011.

Mannucci, A.J., B.D. Wilson, D.N. Yuan, C.H. Ho, U.J. Lindqwister, and T.F. Runge, A global mapping technique for GPS-derived ionospheric total electron content measurements, Radio Sci., 33, 565-582, 1998.

Mannucci, A.J., B.T. Tsurutani, B.A. Iijima, A. Komjathy, A. Saito, W.D. Gonzalez, F.L. Guarnieri, J.U. Kozyra, and R. Skoug, Dayside global ionospheric response to the major interplanetary events of October 29-30, 2003 "Halloween Storms", Geophys. Res. Lett., 32, L12S02, DOI: 10.1029/2004GL021467, 2005

Melbourne, W.G. The Case for Ranging in GPS Based Geodetic Systems. Proceedings of the 1st International Symposium on Precise Positioning with the Global Positioning System, Clyde Goad, Editor. 373-386, 1985.

Mukhtarov, P., D. Pancheva, B. Andonov, and L. Pashova, Global TEC maps based on GNSS data: 1. Empirical background TEC model, J. Geophys. Res.: Space Phys., 118, DOI: 10.1002/jgra.50413, 2013.

Ray, J., New pseudorange bias convention, IGS Mail No. 2744, IGS Central Bureau Information System, 2000.

Ray, J., Updated P1-C1 pseudorange bias corrections, IGS Mail No. 3160, IGS Central Bureau Information System, 2001.

Sardón, E., A. Rius, and N. Zarraoa, Estimation of the transmitter and receiver differential biases and the ionospheric total electron content from GPS observations, Radio Sci., 29 (3), 577-586, 1994.

Sardón, E., and N. Zarraoa, Estimation of total electron content using GPS data: How stable are the differential satellite and receiver instrumental biases? Radio Sci., 32, 1899-1910, 1997.

Sotomayor-Beltran, C., C. Sobey, J.W.T. Hessels, G. de Bruyn, A. Noutsos, et al., Calibrating high-precision Faraday rotation measurements for LOFAR and the next generation of lowfrequency radio telescopes, $A \& A, \mathbf{5 5 2}, \mathrm{A} 58$, DOI: 10.1051/0004-6361/201220728, 2013.

Schaer, S., W. Gurtner, and J. Feltens, IONEX: The IONosphere Map EXchange Format Version 1, Proceedings of the 1988 IGS Analysis Centers Workshop, ESOC, Darmstadt, Germany, February 9-11, 233-247, 1998. 
Tsagouri, I., A. Belehaki, N. Bergeot, C. Cid, V. Delouille, et al., Progress in space weather modeling in an operational environment, J. Space Weather Space Clim., 3, A17, DOI: 10.1051/swsc/2013037, 2013.

Weber, G., D. Dettmering, and H. Gebhard, Networked Transport of RTCM via Internet Protocol (NTRIP). A Window on the Future of Geodesy, Springer, Berlin, ISBN (Online) 978-3540-27432-2. Proceedings of the International Association of Geodesy General Assembly, F. Sanso, Editor. 128, 60-64, 2005.
Wild, U., Ionosphere and geodetic satellite systems: permanent GPS tracking data for modelling and monitoring. Geodatischgeophysikalische Arbeiten in der Schweiz, vol. 48, Schweizerische Geodatische Kommission, Ph.D. thesis, 1994.

Wübbena, G., Software developments for geodetic positioning with gps using TI 4100 code and carrier measurements. Proceedings First International Symposium on Precise Positioning with the Global Positioning System, Clyde Goad, Editor. US Department of Commerce, Rockville, Maryland, 403-412, 1985.

Cite this article as: Bergeot N, Chevalier J-M, Bruyninx C, Pottiaux E, Aerts W, et al.: Near real-time ionospheric monitoring over Europe at the Royal Observatory of Belgium using GNSS data. J. Space Weather Space Clim., 2014, 4, A31. 